\section{Title:}

Author(s):

Submitted to:
Supernova Explosions and Hydrodynamical Instabilities: From Core Bounce to 90 Days

Willy Benz, University of Arizona Stirling Colgate, Los Alamos National Laboratory Marc Herant, University of California, Santa Cruz

Proceedings of the CNLS Conference "Modeling the Forces of Nature"

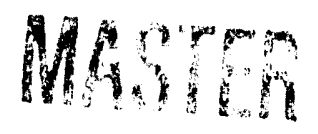

DISTRIEUTION OF THIS DOCUMENT IS UNLIMITED

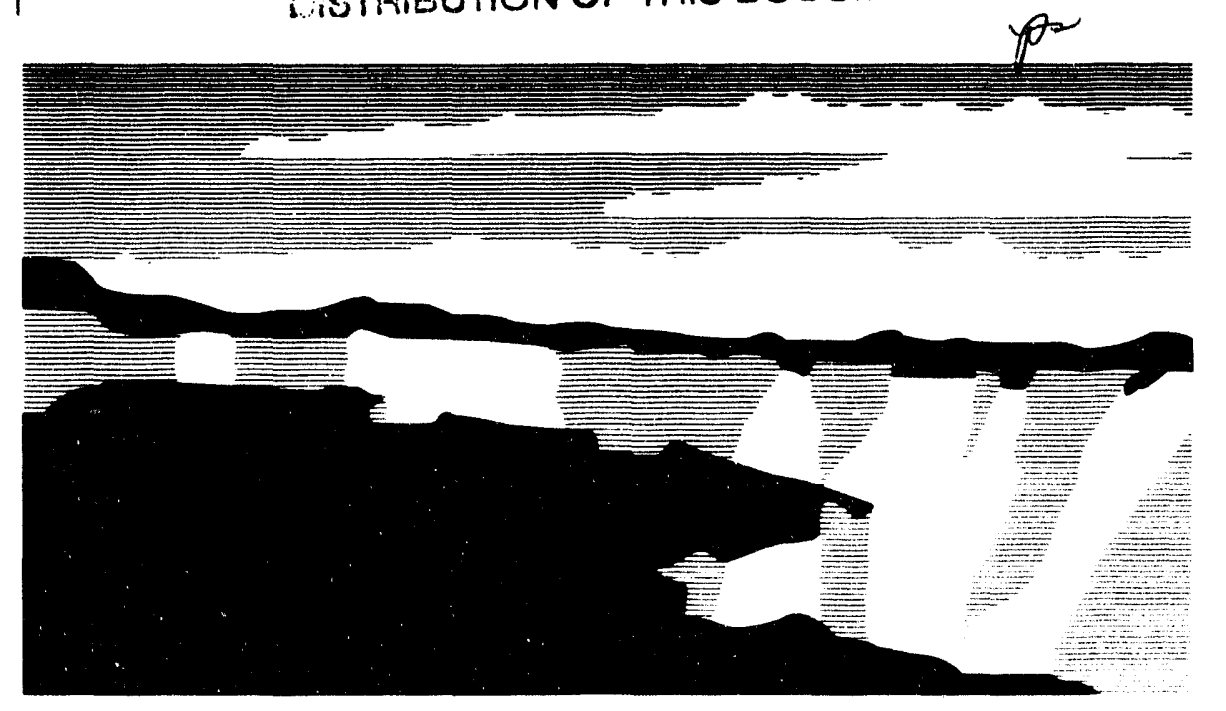

Los Alamos National Laboratory, an affirmative action/equal opportunity empldyer, is operated by the University of California for the U.S. Department of Energy under contract W.7405-ENG-36. By acceptance of this article, the publisher recognizes that the U.S. Government retains a nonexclusive, royalty-free license to publish or reproduce the published form of this contribution, or to allow others to do so, for U.S. Government purposes. The Los Alamos National Laboratory requests that the publisher identify this article as work performed under the auspices of the U.S. Department of Energy. 


\title{
Supernova Explosions and Hydrodynamical Instabilities: Frorn Core Bounce to 90 Days
}

Willy Benz

Steward Observatory. University of Arizona, Tucson, AZ85721

Stirling A. Colgate

Theoretical Division, Los Alamos National Laboratory, Los Alamos, NM 87545

Marc Herant ${ }^{1}$

Lick Observatory, University of California at Santa Cruz, Santa Cruz, CA 95064

\begin{abstract}
:
Since the advent of SN 1987A considerable progress has been made in our understanding of supernova explosions. It is now realized that they are intrinsically multidimensional in nature due to the various hydrodynamical instabilities which take place at almost all stages of the explosion. These instabilities not only modify the observables from the supernova, but are also thought to be at the heart of the supernova mechanism itself, in a way which guarantees robust and self-regulated explosions. In this paper, we review these instabilities placing them into their appropriate context and identifying their role in the genesis of core collapse supernovae.
\end{abstract}

1 Gamma Ray Observatory Fellow

Preprint submitted to Elsevier Science Publishers

8 January 199.3 


\section{Introduction}

From its beginnings in the 1960s, the theory of supernova explosions from stellar collapse has been particularily slow to converge to a generally accepted solution (see [7] and [34] for reviews of early work). For a long time, calculations appeared to oscillate between failed explosions and marginally successful ones dependent on fine-tuning of physical parameters. That some massive stars explode was known, that their core collapses to a neutron star was also accepted, but the connection between the two remained elusive. SN 1987A brought about a radical change in the perception of supernova explosions, for there was no escape from the observational evidence (see [19] for a summary) that extensive mixing, and hence significant departure from spherical symmetry had to occur early in the explosion. The sudden realization that these large departures could only be due to hydrodynamical instabilities early in the explosion combined with increased computer capabilities, provided new incentives to apply hydrodynamical codes to multidimensional studies of supernova explosions.

Large scale instabilities have first been predicted to occur behind the outward moving shock a long time prior to the explosion of SN 1987A ([12,4]. After SN 1987A, they have correctly been identified [6] and modeled by two-dimensional codes [3,17] as RayleighTaylor (RT) instabilities occurring behind the outward moving shock at the various chemical interfaces corresponding to the different burning stages of the progenitor star. These studies did not address the explosion mechanism per say, but relied on initial conditions which artificially assumed an explosion of the appropriate energy, and then investigated the propagation of the explosion shock through the envelope of the star. The amount of mixing induced by these instabilities, although significant, was rapidly found to be insuffcient to explain the extent of the mixing observed in SN 1987A. Subsequently Herant and Benz $[18,19]$ modeled the evolution of the ejecta further in time to examine the dynamical

effects of the ${ }^{56} \mathrm{Ni}$ and ${ }^{56} \mathrm{Co}$ radioactive decay. They also examined the importance of the 
progenitor structure and dimens:onality (three versus two dimensions) of the calculations for the mixing. The main conclusion was that the observations could not be reconciled with the results from the simulations unless a first round of mixing occured in the very early stages of the explosion, close to the time when nickel is synthesized by explosive nucleosynthesis. What better place to point a finger than to the problematic explosion mechanism? And thus, the focus came back to the explosion mechanism itself, with the hope that the late time observations of SN 1987A would provide additional clues to help resolve this enduring problem.

During the 25 years preceding SN 1987A, most of the work on the explosion mechanism had proceeded with one-dimensional calculations of the collapse of the core, and subsequent evolution. This modus operendi had naturally lead to a thinking shaped by a one-dimensional picture of the phenomenon and one wonders how long it would have taken to go beyond it without SN $1987 \mathrm{~A}$ ! Although it was realized that hydrodynamical instabilities would break the spherical symmetry of the problem, most imagined them as operating on small scales, within a mixing length type of framework amenable to a one-dimensional treatment. Epstein [16] was the first to point out potential instabilities induced by $Y_{e}$ gradients in the collapsed core, and those were later taken up by Wilson and Mayle [31] in the doubly diffusive, neutron rich fingers picture. Burrows [8] argued that, as the bounce shock stalls because of neutrino losses and iron dissociation, the postshock entropy decreases creating a region unstable to convection behind the stalled shock. Bethe [7] clearly stated that a neutrino driven explosion would necessarily be convective in nature. However, all these instabilities were principally considered in the mixing-length limit. A notable exception to this trend toward a spherically symmetric picture can be found in the work of Colgate and Petschek [13], Livio, Buchler and Colgate [25], and Smarr et al. [28] investigating the possibility of low order core overturn due to the lepton gradient. Others (e.g. Arnett [2]) were certainly aware of the potential for a fundamental break in the spherical symmetry, but they did not pursue it in detail. 
Motivated mainly by the discrepancy between the observed and calculated ${ }^{56} \mathrm{Ni}$ distribution in SN 1987A, Herant, Benz, and Colgate [20] modeled a simplified, two-dinıensional version of a neutrino driven supernova explosion. That study led to severa! onclusions; (i) strong convective instabilities develop between the neutron star surface and the stalled shock as material is heated from below by neutrino energy deposition, (ii) convection takes place on large scales and breaks the spherical symmetry of the problem, (iii) convection provides an efficient mean to collect energy and transport it from the vicinity of the neutron star to the shock. Taken together, these three conclusions led Herant $\epsilon i$ al. [20] to speculate that multidimensional effects are key to the success of the explosion. Subsequent to this work, Burrows and Fryxell [9] investigated the instabilities related to the decreasing entropy profile set behind the stalling bounce shock. They were able to show that violent convection develops in a time scale of 10 milliseconds, with potentially large effects on neutrin . minosities, and thus on neutrino driven explosions. Their results were later confirmed by the work of Janka and Müller [23].

Although most of the instabilities had by now clearly been identified, each one was still numerically examined in isolation from the others using initial conditions that were not really always self-consistent. Most recently Herant et al. [21] were able to perform comprehensive calculations of stellar collapse and the ensuing explosion in two dimensions, with realistic physics. As in [20], it was found that convection is of crucial importance to the success of the explosion. Guided by these numerical results, a new paradigm for the explosion mechanism was introduced. A supernova is viewed as an open cycle thermodynamic engine in which a reservoir of low-entropy matter (the envelope) is thermally coupled and physically connected to a hot bath (the protoneutron star) by a neutrino flux, and by hydrodynamic instabilities. Neutrino heating raises the entropy of matter in the vicinity of the protoneutron star until buoyancy carries it to low density, low temperature regions at larger radii. This matter is replaced by low-entropy downflows with negative buoyancy. We argue in secton III that this paradigm yields a robust and self-regulated solution to 
the problem of the explosion mechanism.

Almost seven years after the first detection of SN 1987A, supernovae are now seen as harboring hydrodynamical instabilities at almost every stages of their explosion. The image of an expanding "onion-like" layered shell has been abandonned for a much more turbulent and mixed picture of the ejecta. The role of hydrodynamical instabilities has been recognized as important, if not determining, in the inception of successful explosions as well as in the subsequent modification of the observables. In this paper, we try to present a coherent picture of the evoluticn of supernovae which incorporates the recent developments in supernova theory. In order to provide our readers with a road-map to the complex genesis of core-collapse supernovae, we have made extensive use of a flowchart (Fig. 1) which integrates in a logical way most of the important events which occur during the explosion. In sections II, III, and IV, we describe the three, fairly well delineated, main epochs of the explosion, which we have modeled in two dimensions using the so-called Smooth Particle Hydrodynamics (SPH) method. We detail the nature of each hydrodynamic instability and its impact on the overall course of the supernova explosion. We also illustrate each instabilities with figures obtained from our simulations of the problem. The main goal of this paper is to present our perspective of the current state of supernova theory. A detailed description and discussion of all the input physics and of all the numerical aspects of the simulations can be found in Herant et al. $[21,20]$, and Herant and Benz $[18,19]$. We conclude in $\mathrm{V}$ with a brief summary and some comments about tuture avenues of investigation.

\section{Early Instabilities}

Beginning at the top of the flow-chart (Fig. 1), core collapse starts as pressure support is lost because electrons are captured by protons emitting neutrinos and iron photodisin- 
tegrates (an endothermic reaction). The collapse continues even though neutrinos become trapped in the core since a $\gamma=4 / 3$ ratio of specific heat coefficients is too small to stop the infall. Since the collapse proceeds inside out (similar to the standard picture of star formation), the collapsing region is divided into two distinct domains: a homologously contracting, subsonic, core, and a mantle infalling at supersonic (nearly free-fall) velocities. As the homologous core reaches a supernuclear density $\left(\rho>10^{14}\right)$ nuclear forces lead to $\gamma>2$ and the collapse halts. A shock forms at the interface with the supersonic mantle, and is further imparted energy by the slight rebound of the homologous core as it settles in an equilibrium configuration. The bounce shock moves out but because the postshock temperature is high, the shock looses energy in dissociating the iron in the infalling mantle, and in neutrino losses. The bounce shock thus quickly stalls and becomes an accretion shock. It is unable to trigger an explosion by itself, as was suggested by the so-called prompt explosion model.

Up to the stall of the bounce shock the evolution of the core is generally believed to be a one-dimensional problem. However, as pointed out by Arnett [1] explosions taking place in the infalling oxygen shell seconds after the beginning of the collapse, could already lead to local instabilities, thus the spherical symmetry could already be broken before core bounce. Further work is required to investigate these issues. Nonwithstanding these effects and in absence of rotation, the collapse appears to proceed in a spherically symmetric fashion until a few milliseconds post-bounce. The situation changes dramatically after the shock stalls. As indicated by the flowchart (Fig. 1), two separate unstable regions appear. The evolution which was until then one-dimensional develops an intrinsically multidimensional character, and from then on, the results from one-dimensional calculations loose their physical meaning. As we shall see, these two unstable regions are located at the edge and outside of the neutrinosphere. 


\subsection{Ye Gradient Driven Ir stabilities}

The outer edge of the protoneutron star $(r \sim 40 \mathrm{~km})$ is deleptonized much more rapidly than the inside because it can almost freely radiate neutrinos away. This results in a negative lepton abundance gradient which is unstable under the action of the gravitational

field of the protoneutron star (this comes from $P_{l} \propto Y_{l}^{4 / 3}$ in some regimes, see [16] for details). In essence, this situation is analogous in character to Rayleigh-Taylor instabilities driven by differences in molecular weight (as opposed to entropy). To some extent the instability can be stabilized by a positive entropy gradient, but as shown by Wilson and Mayle [31], this would still lead to a so-called doubly diffusive instability.

The lepton-driven instability, which develops over a timescale of about $10 \mathrm{~ms}$, is confined to the relatively narrow range of radii where the neutrino mean free path increases suddenly to macroscopic lengths. As a result, the unstable region is only $15 \mathrm{~km}$ wide and is located at a radius of about $40 \mathrm{~km}$ (Fig. 2). Because of this relatively large aspect ratio, the number of convective cells is not as small as in entropy driven convection (see below); $\sim 5$ in a $90^{\circ}$ two-dimensional cylindrical geometry calculation. Throughout the simulation, the intensity of the convection is variable reaching maximal turn-over velocities of $4000 \mathrm{~km} \mathrm{~s}^{-1}$, while at other times, the velocity can decrease below $1000 \mathrm{~km} \mathrm{~s}^{-1}$. This is probably due to the fact that it sensitively depends on the specifics of the neutrino transport.

The main impact of the lepton-driven instability is to boost the early neutrino luminosity from the protoneutron star. This occurs because the advection of neutrinos by the motions of the matter is much more efficient at transporting neutrinos than simple diffusion through the core. Some (Burrows mainly [11] [10]) have argued that this increase in the neutrino luminosity is the key to successful supernova explosions. Although it is clear that a higher neutrino luminosity favors explosions, we do not believe that this factor in itself is sufficient to guarantee robust and seld-regulated explosions. This can be under- 
stood in the following way. Even with an enhanced neutrino luminosity, the timescale for a neutrino driven explosion remains much longer than the sound crossing time. If there were no further instabilities, the system would equilibrate hyarostatically and tend to conne to an emission-absorption radiative equilibrium with the additional neutrino luminosity. An explosion therefore would have to result from small differences between absorption and emission which are sensitive to the details of the physical conditions of the problem [11]. In our minds, supernovae explosions are robust and self-regulated as the narrow range of explosion energy observed clearly indicates. Thus, any proposed explosion mechanism should reflect these properties and should be rather unsensitive to changes in the physical parameters of the models. A test that no models have yet been truly able to pass.

\subsection{Instabilities Associated with the Stalling Shock}

The instability due to the stalling shock develops further out than the lepton-driven instability $(r \sim 50-150 \mathrm{~km})$ and is driven by a negative entropy gradient. At first, this negative gradient is due to the stall of the bounce shock [8]; as the shock weakens, the entropy increment it imparts becomes smaller and smaller further out in radius. Even without any neutrino heating this entropy profile rapidly $(\sim 10 \mathrm{~ms})$ leads to convective motions as the high entropy material located at the bottom of the potential well of the neutron star, rises through lower entropy material above it [9]. Figure 3 illustrates the convective patterns present in the supernova at $15 \mathrm{~ms}$ past bounce. At this early time, neutrino heating is still negligible and all the convective motions seen around $100 \mathrm{~km}$ are due to the negative entropy profile set by the stalling prompt shock.

Because it is located at a radius were the optical depth to neutrinos is quite low, this instability contributes only marginally to the increase of the early neutrino flux. In fact, despite its apparent violence, the main effect of this instability in the subsequent development of the explosion is essentially the seeding of the next round of instabilities 
due to neutrino heating of the bottom of the envelope. As we shall see in the next section, by allowing low entropy material to be in the immediate vicinity of the proto-neutron star early on, this instability sets up an efficient convective thermodynamic engine which is critical in generating robust and self-regulated explosions.

\section{The Convective Engine}

Soon after the beginning of the early convection disscussed above, neutrino heating becomes important and the negative entropy profile is maintained as the material is now heated from below. A gain radius (radius above which neutrino heating is greater than neutrino cooling) is established between the neutron star surface and the stalled shock. As a consequence, the supernova switches smoothly between the two instabilities, the earlier one seeding the next. Already $25 \mathrm{~ms}$ after the core bounce, convection is fully developed and energy is slowly being accumulated inside the envelope.

Figure 4 shows a plot of velocity vectors at $50 \mathrm{~ms}$ after bounce. As seen from this Figure, the mode number of the instability tends to be small, starting out at 2.4 cells in the $90^{\circ}$ wedge covered by our computations and decreasing to one inflow and one outflow as time goes on. This can be understood [14] because, to first order, the density scales as $r^{-3}$ in the convective region. Consequently a bubble rising in such a medium expands in

size (with $\gamma=4 / 3$ ) proportionally to the radius, thus conserving its shape. Such a shape preserving transformation will be entropy conserving provided entrainment (by KelvinHelmoltz shear instabilities) is exceeded by the expansion of the bubble. As a result, the bubble can rise through many pressure scale heights without fragmenting. Therefore the convective flows take place without breaking-up or eddy-stacking between two natural boundaries: the inner boundary lies where the matter begins to be decoupled from the neutrino fields, whereas the outer boundary is set by the position of the shock. 
One should also note that once a parcel of matter has been heated in the vicinity of the neutron star and ejected by buoyancy, it does not come back down unless it is displaced by matter of still higher entropy. This assumes that the parcel never loses its high entropy, Lecause the fractional entrainment is small as it rises, and in addition adiabatic expansion of the rising bubbles reduces their temperature sufficiently to minimize energy losses in neutrino emissions. As long as the explosion is not decisively successful, there is a nearly infinite supply of unheated, low-entropy matter which replaces matter buoyed away. In that sense, the entropy driven convection can be thought of as an "overturn" which takes place just once, contrary to the case of the lepton-driven convection in which a parcel of matter may cycle through several times.

After an amount of time which varies depending on the progenitors and the details of the physics, but which is always of order 50-100 ms after the bounce, the energy buildup in the convective region and the thinning of the infalling envelope allows the shock to decisively move forward. As the shock propagates outward, its progression becomes easier, as it encounters decreasingly dense material with slower infall velocity. By $100 \mathrm{~ms}$ after the bounce, the shock is located about $1000 \mathrm{~km}$ above the neutron star and is able to impart significant velocities (comparable to or larger than escape velocity) to the infalling matter. This effect coupled with the strong decrease of effective gravity, considerably increases the timescale for mass supply to the convect: a engine operating next to the neutron star. By $200 \mathrm{~ms}$ after bounce, a successful explosion of energy larger than one foe has been obtained producing a supernova, independently of further events in the neighborhood of the protoneutron star.

\subsection{How the Convective Engine Produces an Explosion}

Following Herant et al. [21], we view a supernova as an open cycle thermodynamic engine in which a reservoir of low-entropy matter (the envelope) is thermally coupled and 
physically connected to a hot bath (the protoneutron star) by a neutrino flux, and by hydrodynamic instabilities. In essence, a Carnot cycle is established in which convection allows out-of-equilibrium heat transfer driving low entropy matter to higher entropy and thereby extracting mechanical energy from the heat generated by gravitational collapse. The supernova engine is efficient for two reasons. The mechanical efficiency is high because mixing during the heat exchange is limited by the rapid rise and shape-preserving expansion of the bubbles in a $\rho \propto r^{-3}$ atmosphere. The ideal Carnot efficiency is high because of the large temperature contrast between the surface of the protoneutron star and the envelope at larger radii (in spite of shock heating which is relatively small). By direct $P d V$ integration over the convective cycle, we estimate the energy deposition to be $\sim 4$ foes per $\mathrm{M}_{\odot}$ involved. Further, convection, by keeping the temperature low in rising neutrino-heated high-entropy bubbles, allows the storage of internal energy while minimizing the losses due to neutrino emission.

As indicated by the flowchart (Fig. 1), the convective engine is supplied with low-entropy matter until an explosion has been obtained. Indeed, since the protoneutron star maintains a significant neutrino luminosity for several seconds, there is ample tıme to develop an explosion. Thus convection continues to build up energy exterior to the neutron star until the envelope is expelled and therefore uncoupled from the heat source (the neutron star) and the energy input ceases. This paradigm does not invoke new or modified physics over previous treatments, but relies on compellingly straightforward thermodynamic arguments. It provides a robust and self-regulated explosion mechanism to power supernovae which is effective under a wide range of physical parameters. 


\section{Late-Time Instabilities}

The unexpected early detection in mid-August 1987 (six months after SN 1987A was first seen) of X-rays from SN 1987A by the Ginga satellite and the Kvant-Mir space station $[29,15]$ and later of $\gamma$-rays [26] triggered a flurry of multidimensional studies of supernova explosions. All canonical models in fashion at the time (see [19] for a review) indeed predicted that these high energy photons would only be seen about one year after the explosion, when the expansion factor had become large enough for the optical depth to the radioactive cobalt to be small enough.

A general consensus was rapidly reached that the observations could only be explained by assuming that a large amount of mixing had taken place during the explosion. Thus, the radioactive ${ }^{56} \mathrm{Ni}$ which later decays to ${ }^{56} \mathrm{Co}$, the emitter of the high energy photons, would be brought closer to the surface of the ejecta (and hydrogen would be mixed down toward the center) resulting in a smaller optical depth. As mentioned in the Introduction, the first mechanism which was invoked to provide such mixing was the action of RayleighTaylor (RT) instabilities at the chemical interfaces ( $\mathrm{He} / \mathrm{O}$ and $\mathrm{H} / \mathrm{He}$ ) which occur during the propagation of the supernova blastwave through the envelope.

Multidimensional calculations [3,17] showed that the ejecta of SN 1987A was mixed by the RT instabilities, but that those did not involve ${ }^{56} \mathrm{Ni}$ sufficiently to explain the observations by themselves. It was then hoped that the expansion caused by the heating due to the local deposition of radioactive decay energy during the first weeks (when the ejecta was still optically thick) would drive ${ }^{56} \mathrm{Ni}$ (and therefore ${ }^{56} \mathrm{Co}$ ) further out in the ejecta. Once again, this effect was found insufficient [19] to account for the observations.

Having exhausted all these alternatives forced a reexamination of the conditions which lead to the synthesis of ${ }^{56} \mathrm{Ni}$ during the first phase of the explosion. Herant and Benz [19] suggested that the convection associated with the explosion mechanism itself would result in an extensive mixing of the inner metal core and thus allow the subsequent RT 
instabilities to carry some nickel further out. Arnett (private communication) on the other hand, suggested that explosions in the oxygen shell during core collapse would not only result in the production of some nickel but also leave behind large fluctuations that could seed the later instabilities (this has yet to be confirmed by detailed calculations). Which explanation is correct is a pending question, so that we have included both in our flowchart of supernova explosions (Fig. 1). It is however remarkable that observations of the ejecta several hundred days after the explosion have provided important clues on the early phases of the evolution, and led in part to the important developments on the explosion mechanism which have discussed above.

Having described the circuitous historical and physical connections between the observations of SN 1987A and the explosion mechanism, we now proceed with a more detailed presentation of the late-time instabilities.

\subsection{Rayleigh-Taylor Instabilities}

Once a successful explosion shock has been initiated, it propagates through the envelope of the star and only reaches the surface several hours later. Although the density profile in the envelope is roughly $\propto r^{-3}$ overall, the different entropies corresponding to different burning histories of various chemical shells lead to the existence of density jumps at the interfaces of the shells. Moreover, the hydrodgen envelope tends to have a density distribution shallower than $r^{-3}$. As a result, the shock accelerates as it encounters the sharp drops in density at the shell interfaces $(\mathrm{O} / \mathrm{He}, \mathrm{He} / \mathrm{H})$ and sets up decreasing density profiles at those locations. On the other hand, the shock decelerates when it plows through the hydrogen envelope (see Sedov's results for blastwaves in density power laws [27,22]). This slow-down has to be communicated to the ejecta behind the shock by pressure waves, and consequently, denser material feels an acceleration pushing it into low-density material, or in other words, low-density material has to support high density material. 
This leads to a classical Rayleigh-Taylor instability at the interfaces between shells and thus to mixing.

Figure 4 shows the result of a two-dimensional calculation of a supernova explosion 400 minutes into the explosion, after the shock has emersed from the star, and after the driving force of the instability has ceased. The initial conditions for the simulation were obtained by generating an artifical explosion in a realistic progenitor for SN $1987 \mathrm{~A}$ provided by K. Nomoto. The model was evolved in a one-dimensional code for the first 300 seconds, at which time it was mapped into the two-dimensional code. Inspection of Figure 4 reveals that although extensive mixing has occured at the shell interfaces, the nickel which was assumed to be initially located at the bottom of the ejecta, where it is created by explosive nucleosynthesis, has not been carried far out in the envelope. This led to the speculation[19] that premixing in the early phases of the explosion (either by the explosion mechanism, or by Arnett's flashes in the oxygen shell) had to bring the nickel closer to the regions of RT instabilities, thus allowing it to be mixed further out in the ejecta.

It is also noteworthy that in order to get mixing of the magnitude shown on Figure 4 , perturbations of at least $5 \%$ are required in the initial conditions of the two-dimensional simulations. Colgate, Herant and Benz [14] have shown that the time available for the growth of the RT instability only allows a few $e$-foldings, because the shock emerges from the star quickly. Consequently, in order to reach the non-linear regime as is necessary for extensive mixing, large perturbations are required to seed the instability. Here again, the earlier convection and/or explosions in the oxygen shell have been invoked to explain the origin of these perturbations (see flowchart, Fig. 1). Interestingly, had the progenitor of SN 1987A been a red (instead of blue) supergiant as is thought to be the case for classical Type II plateau supernovae, the situation would have been quite different. In red supergiants, the extent of the hydrogen envelope is such that the shock emerges only after a time $\sim \operatorname{day}(\mathrm{s})$, thereby providing a driving force for the RT instabilities for a prolonged 
period. In those supernovae, RT instabilities grow from infinitesimal perturbations to a fully non-linear regime[22].

\subsection{Radioactive decay}

During the first few weeks that follow the explosion, the energy release associated with the radioactive decay of nickel into cobalt and the subsequent decay of cobalt into iron (with respective half-lives 6.1 and 77.8 days), locally heats the material, because the ejecta is still dense enough to be optically thick to gamma rays. For a short period, it was conjectured that additional mixing of nickel further out in the ejecta might occur due to the expansion connected with the radioactive heating. Indeed, it was pointed out by Woosley [33], that the specific energy liberated in these decays is of the same order as the kinetic energy of the flow itself.

Figure 5 shows the structure of the ejecta 90 days into the explosion. A comparison with Figure 4 makes it clear that the release of radioactive energy does not succeed in mixing nickel much further out in the ejecta. Rather, the expansion has made the nickel flow back into the inner regions, creating a giant hot bubble in the center. The expansion crushes part of the RT fingers into small overdense "nuggets" of hydrogen, helium or metals or a combination of them, with nickel occupying most of the volume. However, because nickel was not mixed very far out, these nuggets are confined to the central regions only. We speculate that had the mixing be sufficient, nuggets would be found much further out with nickel filling most of the available volume as is indicated by the analysis of observational data from SN 1987A by Li, McCray and Sunyaev [24].

The simulation was performed with the assumption that the radioactive energy is deposited locally, in the nickel, in the form of heat. Although this is correct for a large amount of the time covered by the calculation, the assumption breaks down toward the end (after most of the energy has been released anyway) as the expansion of the ejecta 
makes it optically thin. If anything, the calculation therefore overestimates the effects of the radioactive energy input. Although the radioactive decays are clearly important for the evolution of the structure of the remnant, they can be ruled out as the main cause for the mixing of nickel far out into the ejecta, thus once again pointing to the early instabilities which were discussed above (Fig. 1).

\section{Conclusions}

Since SN 1987A, multidimensional modeling of supernova explosions has proceeded at an accelerated pace. In this paper, we have tried to present an overview of hydrodynamical instabilities in supernovae. From core collapse to about 100 days later, instabilities occur almost continuously. In succession we have $(t=0$ at core bounce $):(t \approx-100 \mathrm{~ms})$ possible explosions in the collapsing oxygen shell; $(t \approx 10 \mathrm{~ms}) Y_{e}$ gradient driven instability at the edge of the protoneutron star; $(t \approx 15 \mathrm{~ms})$ unstable entropy gradient set by the stalling bounce shock, $(t \approx 25-200 \mathrm{~ms})$ convection driven by neutrino heating - the convective engine; $(t \approx$ hours) RT instabilities at the chemical shell interfaces due to the propagation of the shock through the envelope; $(t \approx$ weeks) expansion of a hot nickel bubble due to the radioactive energy input from the decay of ${ }^{56} \mathrm{Ni}$ and ${ }^{56} \mathrm{Co}$. As the flowchart shows (Fig. 1), all these events are interconnected and one stage influences the next. Conversely, this has permitted late time observations of SN 1987A to provide clues about the first stages of the explosion.

Emerging from this numerical work is that these instabilities tend to occur on large scales, and thus one-dimensional models cannot account for their dynamical importance well. In retrospect, we believe that a lot of the controversy surrounding the work on the supernova explosion mechanism probably stemmed from the use of numerical tools that

did not capture the essence of the phenomenon because they assumed spherical symmetry. 
Since one-dimensional codes can only handle hydrodynamic instabilities through the use of artificial prescriptions, different implementations of these numerical recipes have lead to different, sometime questionable, answers.

Instabilities occurring within the first 100-200 milliseconds following core bounce are probably responsible for the success of the explosions. By taking the system away from a quasi-static, emission-absorption equilibrium, these particular instabilities render the neutrino powered supernova explosions insensitive to the fine-tuning of the nuclear and neutrino physics. Explosions therefore occur over a wide range of physically sensible input parameters. Furthermore, by supplying cold, low-entropy material to the vicinity of the protoneutron star, these instabilities ensure a high energy deposition efficiency while dramatically cutting down losses by allowing high entropy, bouyant eddies to rise before they radiate their energy content away in neutrinos. This process continues until the supply of new material brought down toward the neutron star dwindles, as a successful explosion gets underway. Instabilities following core bounce therefore achieve naturally what no other paradigm of supernova explosions has provided so far: a robust and self-regulated mechanism that guarantees explosions of the appropriate magnitude.

For practical reasons, most simulations to date have focused on specific stages of the explosion. Although this allows to gain insight into some aspects of the problem, it lacks in comprehensiveness and could potentially lead to erroneous conclusions, for instabilities feed on each other, the first one seeding the next, and so on. By studying instabilities in isolation, not the global picture is lost, but also the proper initial conditions. Recent multidimensional simulations[21] which follow the supernova from the onset of core collapse to to several hundred milliseconds after bounce are a step in the direction of a more comprehensive approach, free from the numerical artifacts associated with remapping. However, the seamless modeling of a supernova from collapse to remnant is an objective still to be attained, but currently pursued vigorously by several groups.

Other aspects of multimensional hydrodynamics in supernovae promise to present im- 
portant challenges for years to come. So far most simulations have been performed in two dimensions rather than three for practical computing reasons. While we expect that the main features of the instabilities will be preserved in three dimensions [19], this needs to be checked. In this paper, we have ignored events around the protoneutron star after a successful explosion has been launched. It is probable that a significant amount of material from the ejecta falls back on the protoneutron star, with potential instabilities of importance for issues such as r-process nucleosynthesis[32]. Finally, the expansion of the remnant into the interstellar medium will certainly trigger instabilities in addition to those due to the non-uniform radiative cooling of the now transparent ejecta. A long term objective of supernova multidimensional hydrodynamics is to connect the structure of remnants for which spatially resolved observations are available to the explosion process.

\section{Acknowledgement}

This work has benefited from stimulating discussions with Dave Arnett, Hans Bethe, Adam Burrows and Stan Woosley. The work by W.B. was supported in part by NSF grant AST-0206378. The work of S.A.C was supported by the Department of Energy and M.H. was supported by a fellowship from the Compton Gamma Observatory.

\section{References}

[1] Arnett, D. in IAU Colloquium 145, Supernovae, Xian, China (1993)

[2] Arnett, D. in IAU Symposium 125, The Origin and Evolution of Neutron Stars, eds. Helfand, D. J., and Huang, J.-H., (Dordrecht: Reidel) (1987) 273

[3] Arnett, D., Fryxell B., and E. Müller Astrophys. J 341 (1989) L63. 
[4] Bandiera, R. Astron. Astrophys. 139 (1984) 368.

[5] Baron, E. A. and J. Cooperstein Astrophys. J. 353 (1990) 597.

[6] Benz, W., and F.-K. Thielemann Astophys. J. Letters, 348 (1090) L17.

[7] Bethe, H. Supernova mechanisms. Rev. of Mod. Phys. 62 (1990) 801.

[8] Burrows, A. Astrophys. J 318 (1987) L57.

[9] Burrows, A., and B. Fryxell Science 258 (1992) 430.

[10] Burrows, A., and B. Fryxell Astrophys. J. 418 (1993) L33.

[11] Burrows, A., and J. Goshy Astrophys. J. 416 (1993) L75.

[12] Chevalier, R. A. Astrophys. J. 207 (1976) 872.

[13] Colgate, S.A., and A.G. Petschek Astrophys. J. 236 (1980) L115.

[14] Colgate, S.A., Herant, M., and Benz, W. Phys. Rep. 227 (1993) 157.

[15] Dotani, T. et al. Nature 330 (1987) 230.

[16] Epstein, R. Mon. Not. R. astr. Soc. 188 (1979) 305.

[17] Hachisu, I., Matsuda, T, Nomoto, K., and T. Shigeyama Astrophys. J. 358 (1990) L57.

[18] Herant, M., and W. Benz Astrophys. J. 370 (1991) L81.

[19] Herant, M., and W. Benz Astrophys. J. 387 (1992) 294.

[20] Herant, M., Benz W., and S.A. Colgate Astrophys. J. 395 (1992) 642.

[21] Herant, M., Benz W., Hix, R., Fryer, C., and S.A. Colgate Astrophys. J. submitted (1994).

[22] Herant, M., and S.E. Woosley Astrophys. J., in press (1994)

[23] Janka, H.-Th., and E. Müller in Frontiers of Neutrino Astrophysics (Universal Academy Press: Tokyo) (1993).

[24] Li, McCray and Sunyaev

[25] Livio, M., Buchler, R.J., and S.A. Colgate $A$ trophys. J. 238 (1980) L139. 
[26] Matz, S.M., Share, G.H., Leising, M.D., Chupp, E.L., Vestrand, W.T., Purcell, W.R., Strickman, M.S., Reppin, C. Nature 331 (1988) 416.

[27] Sedov. L. I., Similarity and Dimensional Methods in Mechanics (Academic: New Y'ork) (1956).

[28] Smarr, L., Wilson, J. R., Barton, R. T, and R.L. Bowers Astrophys. J. 246 (1981) 515.

[29] Sunyaev, T. et al. Nature 330 (1987) 227.

[30] Wilson, J. in Numerical Astrophysics, ed. J.M. Centrella et al. (Boston: Jores E' Bartlett) (1985).

[31] Wilson, J.R., and R.W. Mayle Phys. Rep. 163 (1988) 63.

[32] Woosley, S.E., Wilson, J. R., Mathews, G. J., Hoffman, R. D., and B. S. Meyer Astrophys. $J$. submitted (1994).

[33] Woosley, S.E. Astrophys. J. 330 (1988) 218.

[34] Woosley, S.E., and T.A. Weaver Ann. Review Astron. Astrophys. 24 (1986) 205.

\section{DISCLAIMER}

This report was prepared as an account of work sponsored by an agency of the United States Government. Neither the United States Government nor any agency thereof, nor any of their employees, makes any warranty, express or implied, or assumes any legal liability or responsibility for the accuracy, completeness, or usefulness of any information, apparatus, product, or process disclosed, or represents that its use would not infringe privately owned rights. Reference herein to any specific commercial product, process, or service by trade name, trademark, manufacturer, or otherwise does not necessarily constitute or imply its endorsement, recommendation, or favoring by the United States Government or any agency thereof. The views and opinions of authors expressed herein do not necessarily state or reflect those of the United States Government or any agency thereof. 


\section{Figure Captions}

Figure 1. Schematic of the most important events affecting a supernova explosion during its first 90 days.

Figure 2. Ye-gradient driven convection at the edge of the nascent neutron star $15 \mathrm{~ms}$ after core bounce (25 M_odot projenitor). Particles are colored according to Ye.

Figure 3. Entropy gradient driven convection $15 \mathrm{~ms}$ after bounce (25 M_odot projenitor). This convection is entirely due to the inverted entropy profile left behind the stalling prompt shock. Notice the Ye-driven convection at smaller radii. Particles are colored according to entropy.

Figure 4. Neutrino driven convection $50 \mathrm{~ms}$ after bounce (25 M_odot projenitor). Cold, low entropy material is supplied to the vicinity of the neutron star ensuring an efficient thermodynamical engine. Notice that the convetive mode has reduced to the minimum mode possible. Particles are colored according to entropy.

Figure 5. Rayleigh-Taylor instabilities behind the outward moving shock 400 minutes after the explosion. These instabilities result in extensive but macroscopic mixing of the ejecta. In this plot blue is hydrogen, green is helium, yellow are metals and red is nickel. The interval between the large tickmarks is $2 \times 10^{7} \mathrm{~km}$.

Figure 6. Structure of the ejecta 90 days after the explosion. Notice how nickel and cobalt decay have modified the structure of the ejecta. Fingers have been crunched into small nuggets and nickel decay products occupy most of the volume. Colors have the same meaning as in Fig. 5. The interval between the large tickmarks is $10^{10} \mathrm{~km}$ 


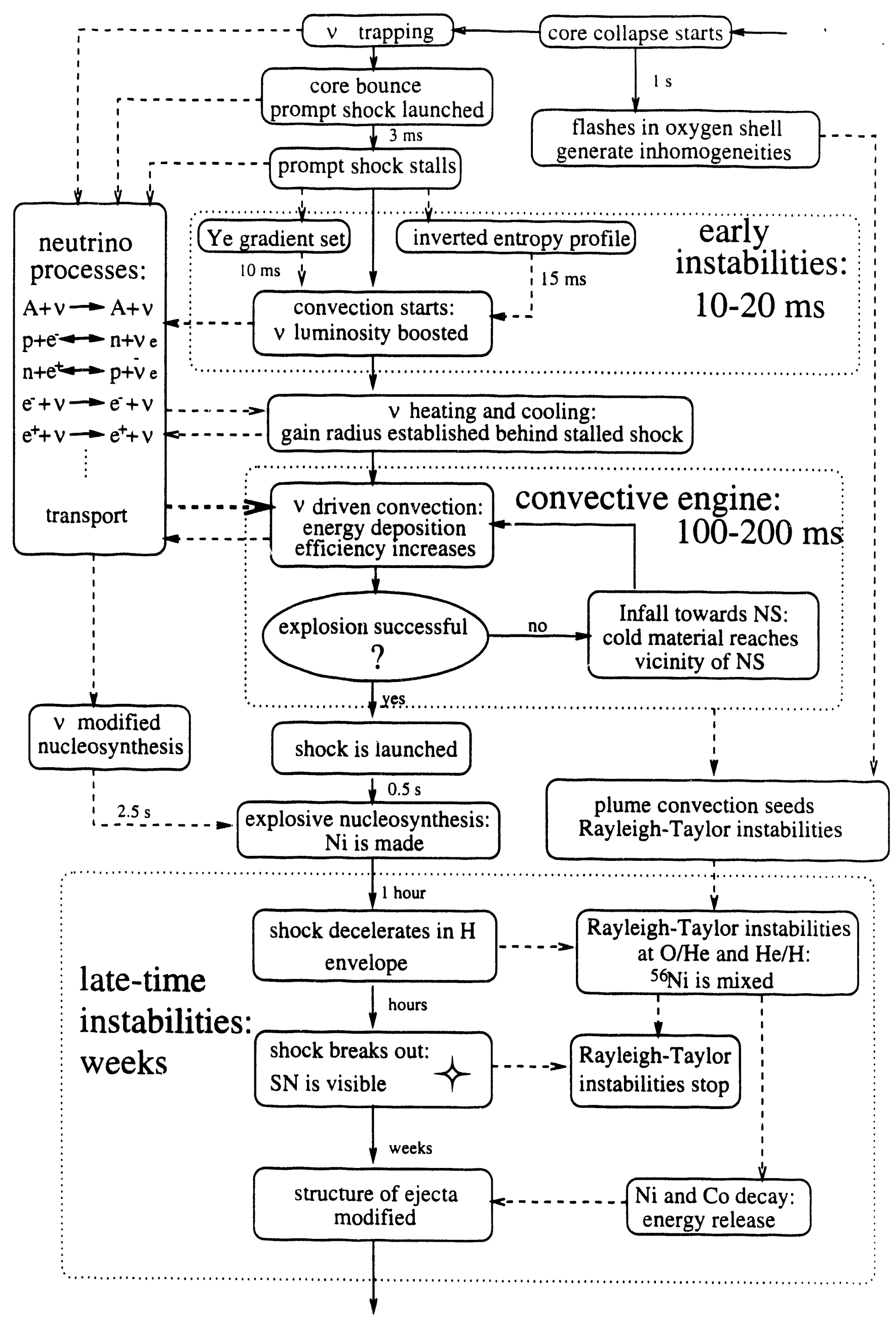




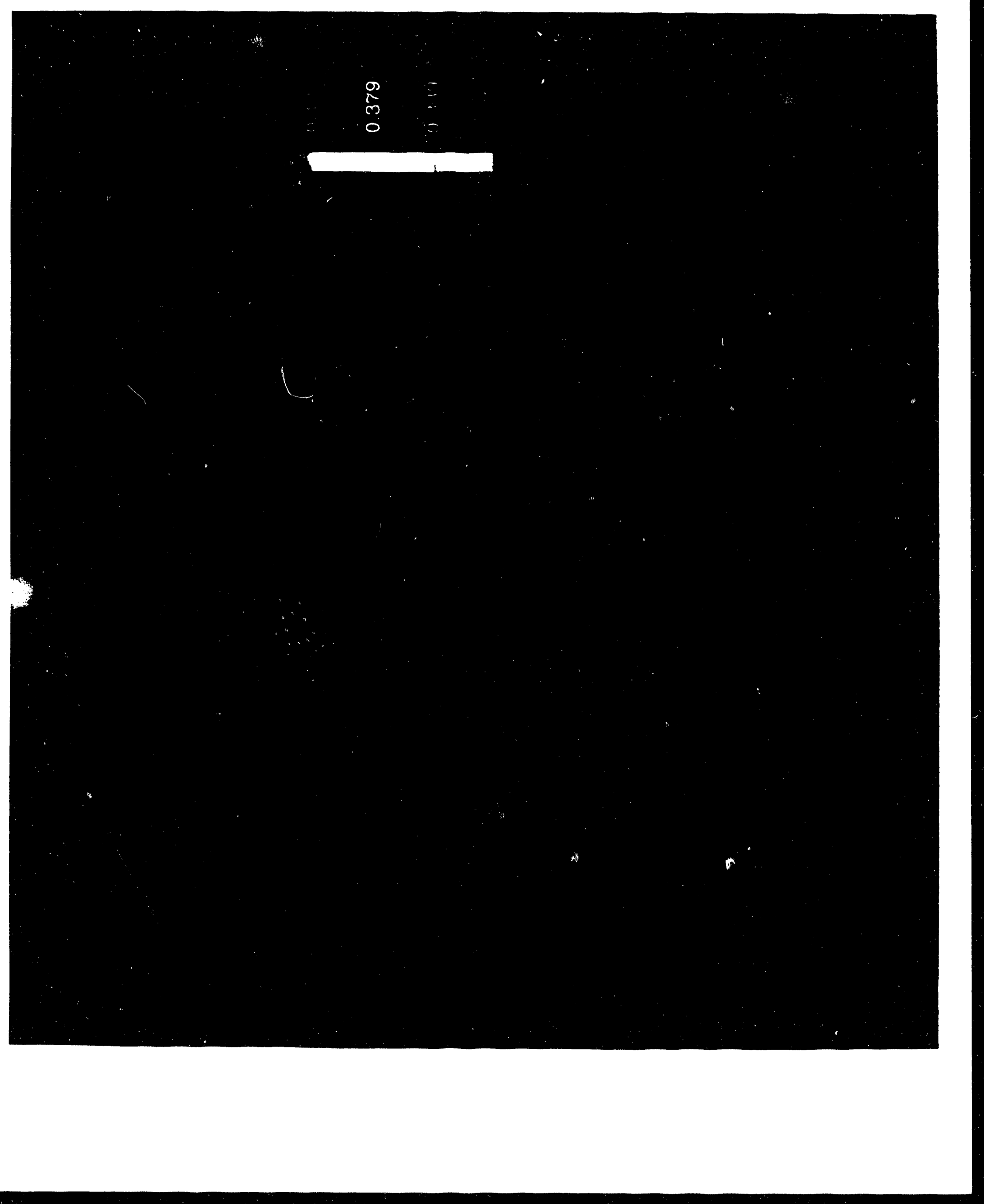




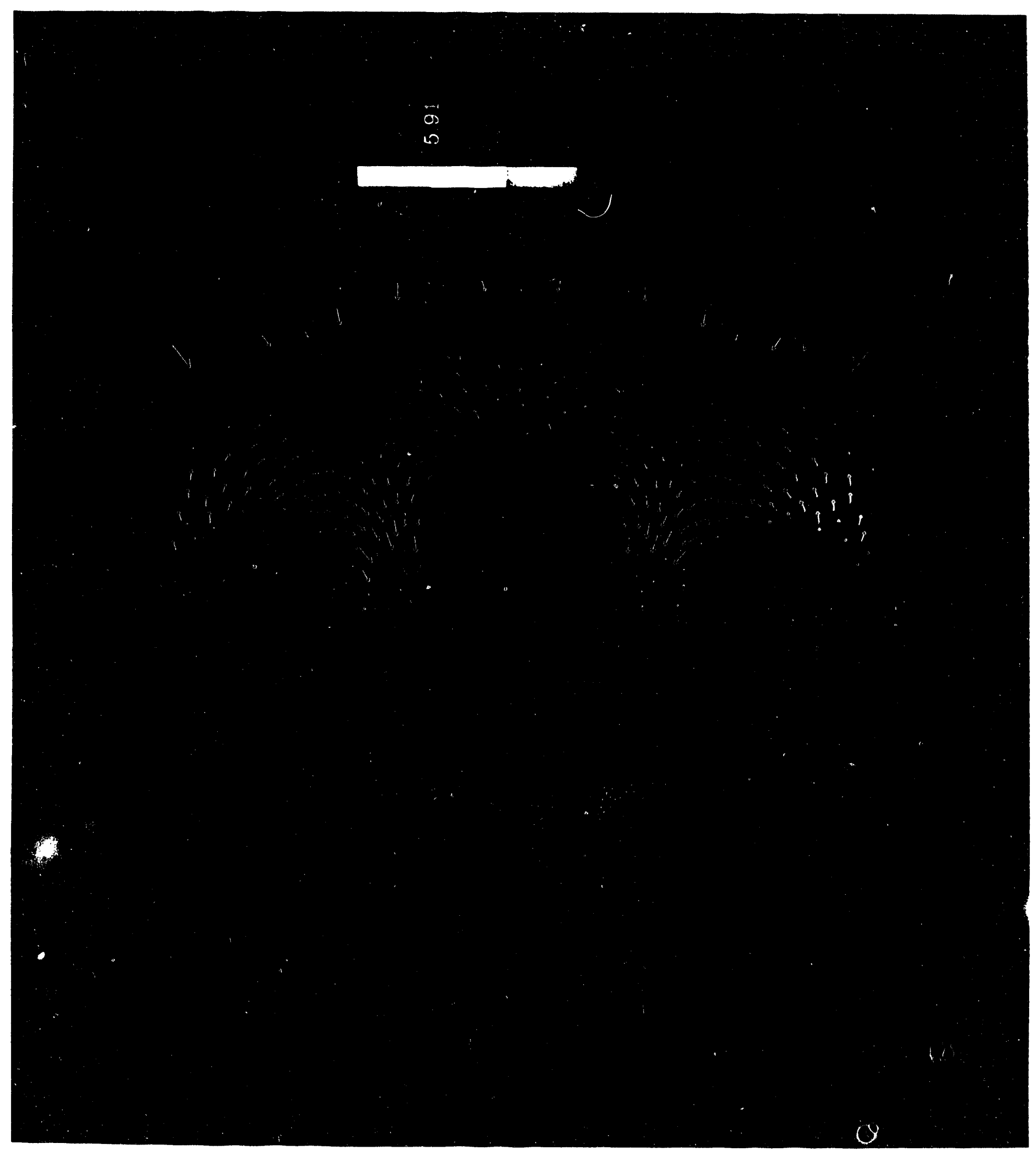




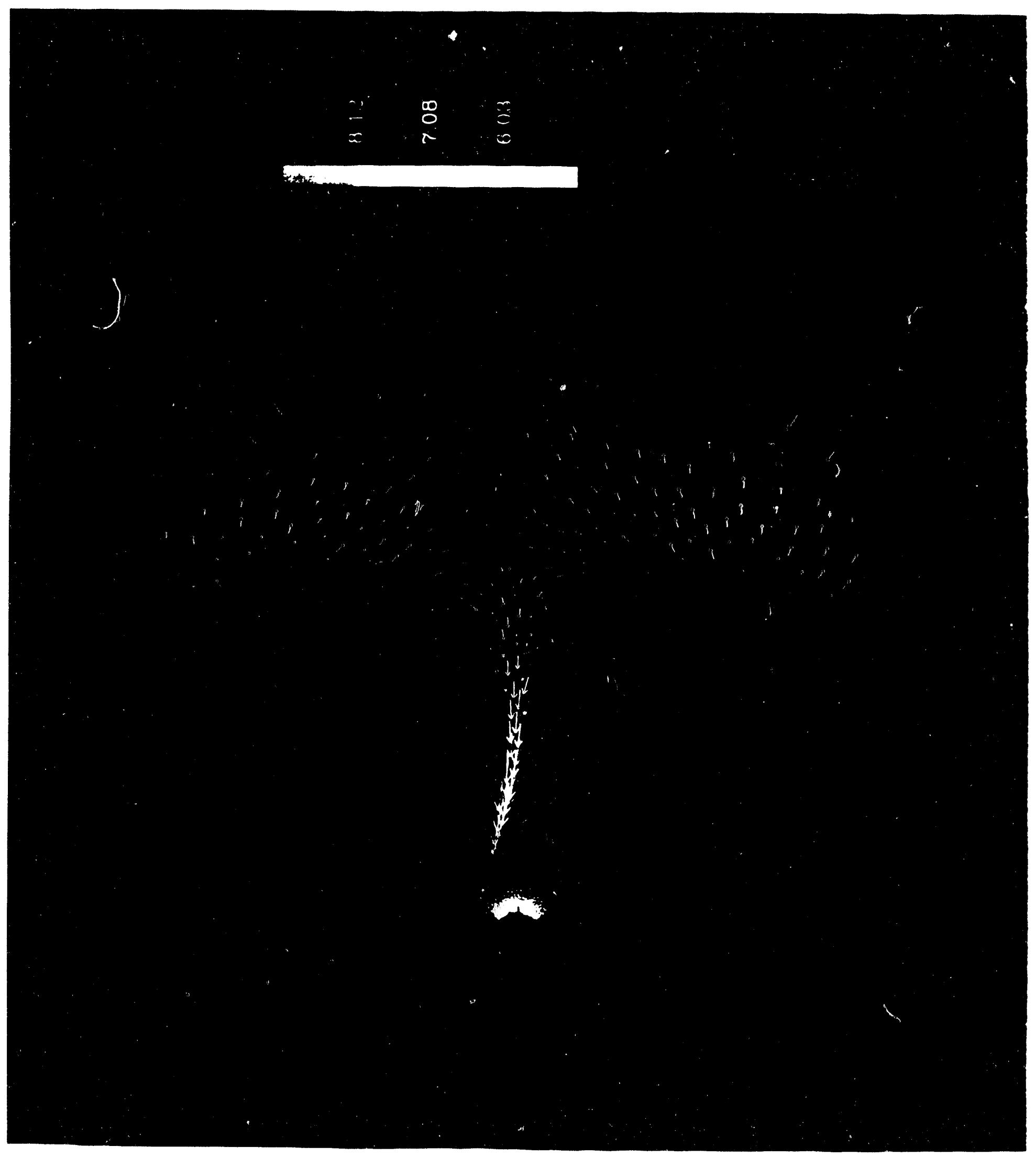


ip

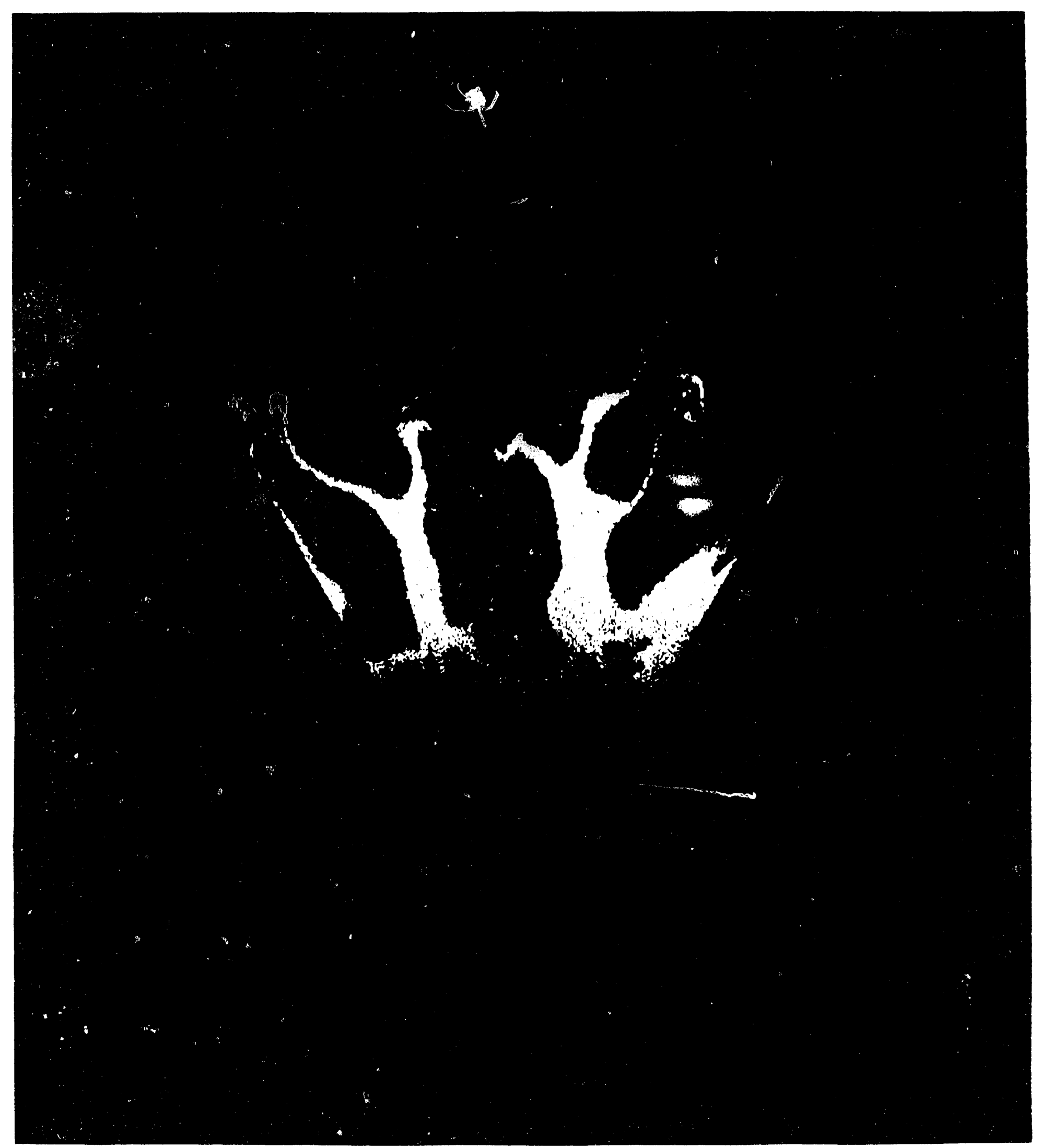




$$
\text { Nit }
$$



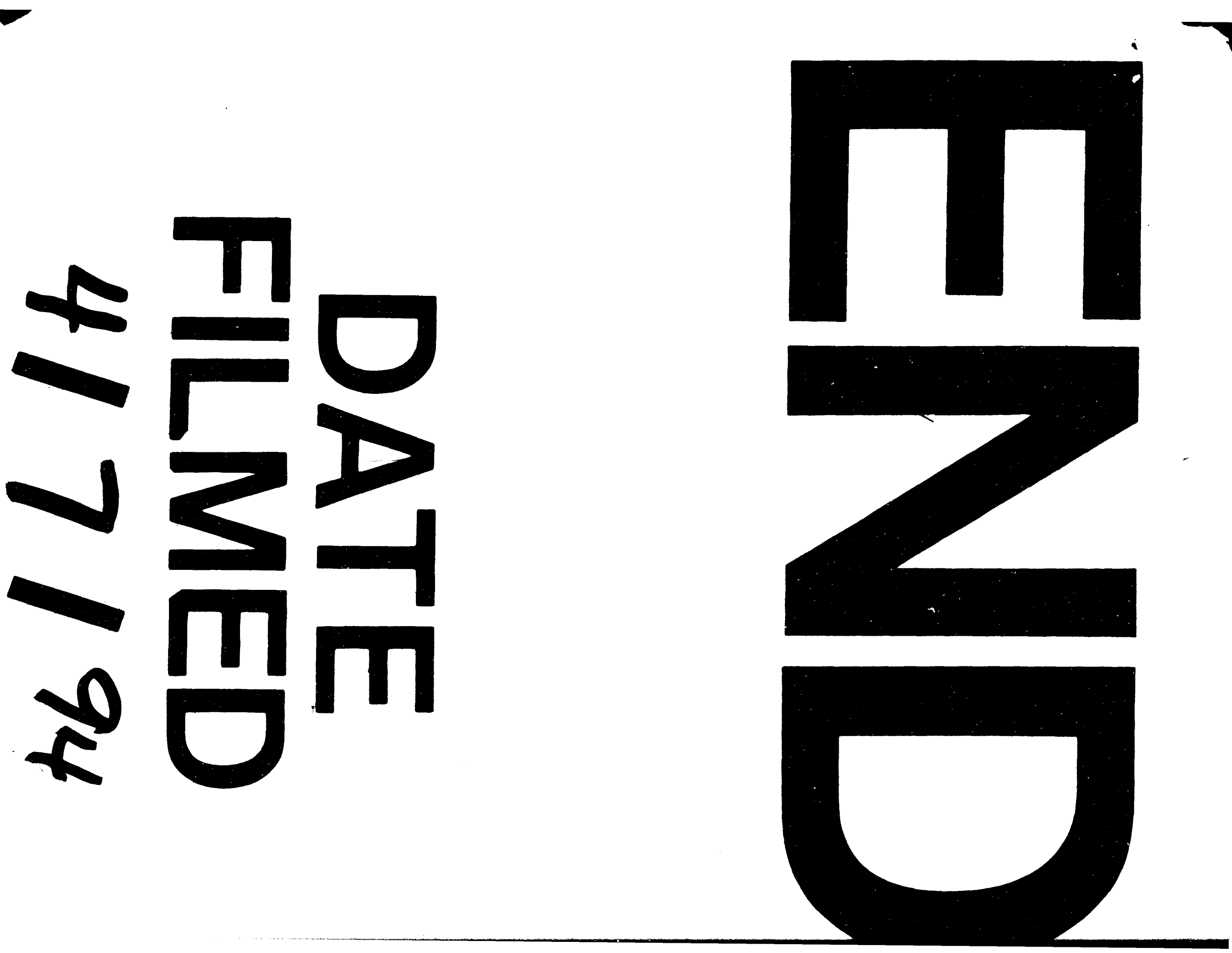